\title{
Tracing the high energy emission of $\gamma$-ray detected radio loud quasars
}

\author{
Giulia Migliori \\ Harvard-Smithsonian Center for Astrophysics, 60 Garden St. Cambridge, MA 02138, USA \\ email: gmigliori@cfa.harvard.edu
}

\begin{abstract}
We present a multiwavelength study of the core and relativistic jet of the radio loud $(\mathrm{RL})$ quasar RGB J1512+020A ( $\mathrm{z}=0.20$ ). We report the discovery of a bright, $13^{\prime \prime}$ extended $\mathrm{X}$-ray jet with a short Chandra observation. We discuss the origin of the jet X-ray emission and its properties in comparison with sample of X-ray quasar jets. The broadband core spectrum is contributed by the emission of the central quasar, by a blazar component, responsible for the $\gamma$-ray emission detected by Fermi, and by the host galaxy. We model the non-thermal blazar spectral energy distribution (SED) and constrain the total jet power. The jet power inferred from the blazar SED modeling is in agreement with the values obtained from the total radio power, pointing to a jet that efficiently carries its power up to kiloparsec scales. The quasar emission appears intrinsically weak in the optical-UV band. The disk luminosity estimated from the broad emission lines is lower than the jet power, in agreement with recent results from observations and theory.
\end{abstract}

Keywords. galaxies: jets, quasars: individual (RGB J1512+020A), X-rays: galaxies

\section{Introduction}

In extragalactic jets, X-ray emission is observed on kiloparsec scales in knots and hot spots (Harris \& Krawczynski 2006). X-ray to $\gamma$-ray radiation is produced in a compact, fast moving region, so called blazar region, within distances of parsec or below from the supermassive black hole $(\mathrm{SMBH})$. The high-energy radiation is a significant fraction of the jet bolometric luminosity, which in turn can be used as an estimate of the minimum power carried by the jet. Modeling of the jet broadband emission allows to infer the main physical parameters, such as its bulk motion, density of the radiating particles and magnetization.

When a $\gamma$-ray blazar component and an X-ray extended jet are both visible in the same object, we can map how the physical parameters change in the jet expansion and investigate its dynamical structure. Estimates of the jet power at the different scales can tell us whether the jet efficiently carries the bulk of the power to its termination. This is also important to understand the impact of the jet on its environment.

In addition, in quasars, the optical-UV emission of the core reveals features related to the accretion process (thermal emission of the accretion disk and broad emission lines). Thus, we can estimate the disk luminosity, the mass of the SMBH and the accretion rate, and measure how the accreted power is divided between the disk and the jet. A recent study of a sample of Fermi detected blazars (Ghisellini et al. 2014) confirms that the jet power correlates with, and is larger than the disk luminosity. According to theoretical models (Tchekhovskoy et al. 2011), this result would point to a primary role of the magnetic field in the jet formation.

In the past, there was a very limited number of X-ray jets associated with $\gamma$-ray detected quasars (see e.g. Tavecchio 2007). Fifteen years of Chandra observations and the launch of Fermi observatory have drastically increased the number of targets for a 

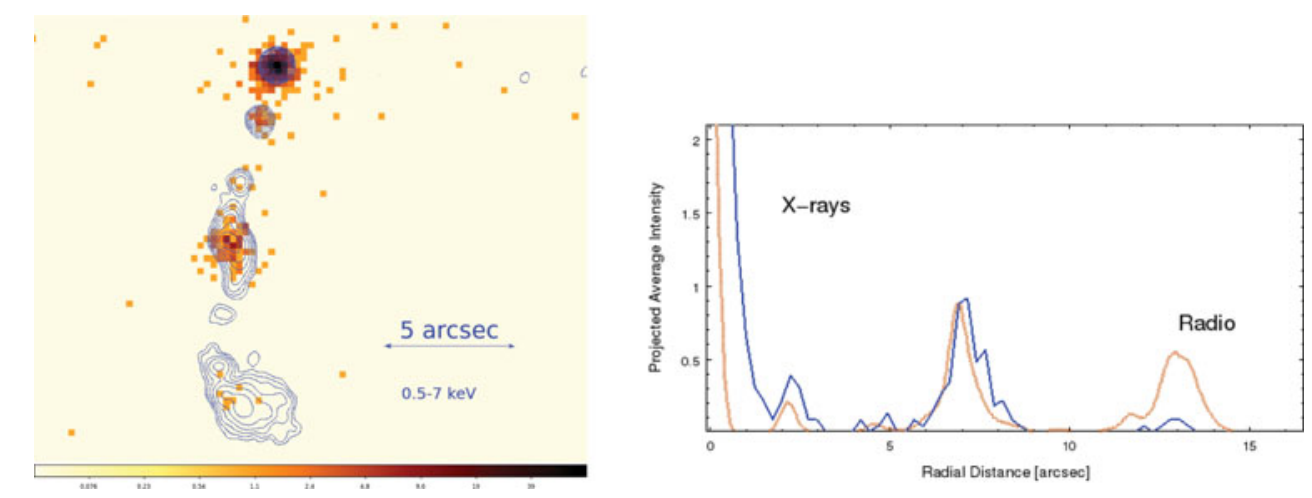

Figure 1. Right panel: Chandra $0.5-7 \mathrm{keV}$ ACIS-S image and overlaid VLA $4.9 \mathrm{GHz}$ contours of RGB J1512+020A. The pixel size is equal to $0.246^{\prime \prime}$. Color scale is logarithmic. The radio map peak is at $0.17 \mathrm{Jy} /$ beam with a restoring beam of $0.45^{\prime \prime}$. The contour levels begin at $0.12 \%$ and increase by a factor of 2 . Extended X-ray emission is clearly visible at the location of the radio jet. Left panel: radio $(4.9 \mathrm{GHz}$, red dashed line $)$ and X-ray $(0.5-7 \mathrm{keV}$, blue solid line) brightness profiles along the jet in arbitrary units.

study of the jet multiscale high-energy emission. Motivated by this, we have selected a sample of radio loud quasars (RL QSOs) in the second Fermi LAT AGN catalog (2LAC, Ackermann et al. 2011) with an extended X-ray jet. The sample covers a redshift range between 0.22 to 1.8 . All the sources have archival Chandra observations. Indeed, a good mutiwavelength coverage, including radio and optical-UV data, is also required to investigate the jet morphology and model the jet and disk emission. The final sample is formed by 14 quasars. Here, we present the analysis of RGB J1512+020A ( $\mathrm{z}=0.20)$.

$R G B J 1512+020 A$ is listed in the Sloan Digital Sky Survey (SDSS) catalog of quasars (Shen et al. 2011). It is a radio source reported in several catalogs and it is associated with the $\gamma$-ray source 2FGL J1512.2+0201 in the 2LAC (Ackermann et al. 2011). In Sbarrato et al.(2012), it is classified as a Flat Spectrum Radio Quasar based on the modeling of the blazar emission. In X-rays it has been detected with ROSAT (Siebert et al. 1998). It was observed with the ACIS camera onboard the Chandra X-ray Observatory for $\sim 3$ ksec on 2005 March 27 (ObsID 5671).

\section{Large scale jet}

The radio map at $4.9 \mathrm{GHz}$ shows an $14^{\prime \prime}$ extended S-shaped luminous jet with a knotty like structure. Extended X-ray emission coincident with the radio jet is clearly visible in the 0.5-7 keV Chandra image in Fig. 1 (left panel). We detected two X-ray knots at $2^{\prime \prime}$ and $7^{\prime \prime}$ distance from the core. A bridge of diffuse emission is present between the two knots and faint X-ray emission $\left(F_{X}=1.6 \times 10^{-14} \mathrm{erg} \mathrm{cm}^{-2} \mathrm{~s}^{-1}\right)$ is detected up to the radio jet termination (at $13^{\prime \prime}$ distance from the core).

Brightest knot - The second knot is the brightest jet component in X-rays, with a $0.5-7$ $\mathrm{keV}$ flux of $2.5 \times 10^{-13} \mathrm{erg} \mathrm{cm}^{-2} \mathrm{~s}^{-1}$. Remarkably, the $0.5-2 \mathrm{keV}$ emission of the knot is $3.4^{\prime \prime}$ extended in the direction orthogonal to the jet main axis, while the hard X-ray (2-7 $\mathrm{keV}$ ) component is unresolved. A deeper Chandra observation is necessary to perform a spatially resolved study of the knot and to understand whether the emission in the two bands is produced by the same process. Note that there are currently only a handful of jets showing a transversally resolved X-ray structure (see 3C 371 and PKS 2201+044, Sambruna et al. 2007). 


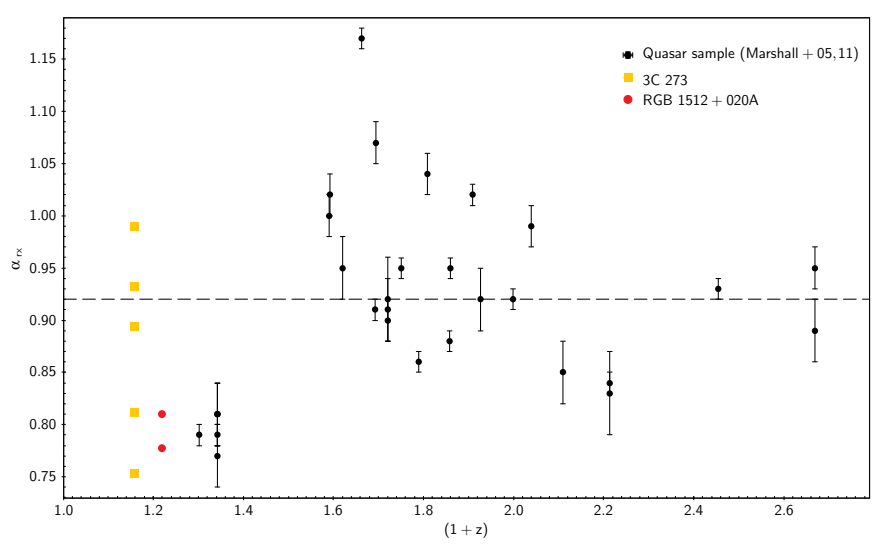

Figure 2. $\alpha_{\mathrm{rx}}$ as a function of the redshift of X-ray detected jet knots: solid red circles are the two knots of RGB J1512+020A jet, black solid circles are the knots of the radio quasar sample observed by Chandra (Marshall et al. 2005,2011, and yellow solid squares are the knots of 3C 273 (Massaro et al. 2011). The dashed line indicates the median $\alpha_{\mathrm{rx}}$ of the quasar sample.

The X-ray luminosity $\left(L_{X}=3.3 \times 10^{43} \mathrm{erg} \mathrm{s}^{-1}\right)$ and spectral index $\left(\Gamma_{X}=1.64 \pm 0.17\right)$, the IC dominated broadband SED and the radio and X-ray brightness profiles (Fig 1, right panel) are typical of jets of powerful Fanaroff Riley II radio sources, whose SEDs are modeled with two components (synchrotron emission for the low-energy emission and IC off microwave background photons, IC/CMB, or high-energy synchrotron emission for the X-rays). However, an extrapolation of the radio emission to the $\mathrm{X}$-rays is also compatible with the current data. Observations in the IR to optical band and polarization are needed to be conclusive on the origin of the X-ray emission (see e.g. Cara et al. 2013 and references therein).

Comparison with X-ray jet samples - The jet of RGB J1512+020A is one of the few $\mathrm{X}$-ray jets associated with a low redshift $(<0.5)$ quasar. Its jet to core X-ray flux ratio is unusually large $(\sim 0.2)$ in comparison with typical values of quasar jets $(\lesssim 0.1$, see Hogan et al. 2011, Marshall et al. 2011 and Massaro et al. 2011). In Fig. 2, we show the radio to X-ray spectral index $\left(\alpha_{\mathrm{rx}}\right)$ of the jet knots of RGB J1512+020A and of the sample in Marshall et al. $(2005,2011)$. The jets located at low redshift, including our source, tend to have the $\alpha_{\mathrm{rx}}$ below the average value found for the sample (marked by the dashed line). Interestingly, in some of these low-z quasars (see 1928+728, 3C273 Sambruna et al. 2004, Jester et al. 2007, respectively), observations would favor the synchrotron scenario over IC/CMB emission for the jet X-ray emission.

\section{Core emission}

$X$-ray core - The Chandra spectrum of the X-ray core emission is well reproduced by a simple power law with a photon index $\Gamma_{\mathrm{X}}=1.71 \pm 0.08$ and no intrinsic absorption required by the data. The $0.5-7 \mathrm{keV}$ flux $\left(1.4 \times 10^{-12} \mathrm{erg} \mathrm{cm}^{-2} \mathrm{~s}^{-1}\right)$ is in agreement with previous ROSAT data and with our new Swift X-ray Telescope (XRT) observations (June-July 2014).

Fermi LAT observations - We considered 6 years of Fermi -LAT observations. The source is clearly detected with a test statistic (TS) of 744 . The $\gamma$-ray spectrum is modeled using a single power-law with a photon index of $2.19 \pm 0.04$ and a measured $0.1-100 \mathrm{GeV}$ flux of $(3.2 \pm 0.3) \times 10^{-8}$ photons $\mathrm{cm}^{-2} \mathrm{~s}^{-1}$. The analysis of the 6 month binned lightcurve shows moderate flux variability (within a factor of 3 ). 


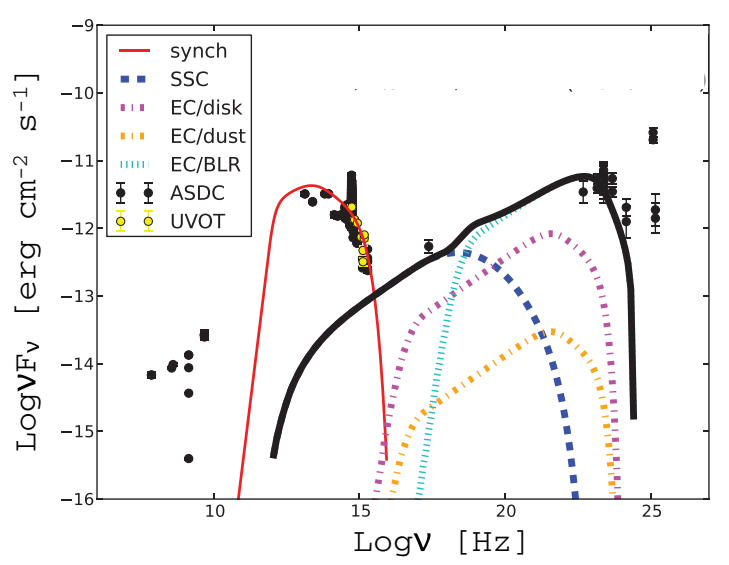

Figure 3. Observed SED and model of the blazar emission. For this model we assumed the following parameter values: $z_{d} \sim 10^{16} \mathrm{~cm}, R \sim 10^{15} \mathrm{~cm}, \theta=4 \mathrm{deg}, \Gamma_{\text {bulk }}=12, \mathrm{~B}=3.3 \mathrm{G}(\theta$, $\Gamma_{b u l k}$ and $\mathrm{B}$ are the jet inclination angle, the bulk motion and the magnetic field, respectively). The magnetic field to radiating electron energy density ratio is $\sim 0.2$. The black thick solid line is the sum of all the IC components.

Optical-UV data - We discovered optical variability between the SDSS spectrum taken in 2001 and our new observation with the Kitt Peak telescope (June 5, 2014). We argue that the flux variation is most likely related to the synchrotron emission of the blazar component based on the fact that: 1- we see the same level of flux variability in optical and $\gamma$-rays; 2 - the equivalent width of the spectral lines decreases with the increasing continuum; 3- the shape of the divided continuum between the two spectra is a power law (with a spectral index $\alpha_{\lambda}=-0.715$ ).

UV data were obtained with the Ultraviolet-Optical Telescope (UVOT) onboard the Swift satellite. We retrieved the available broadband archival data of the core from the SED Builder tool of the ASI Science Data Center (ASDC). The SED with the full dataset is shown in Figs. $3 \& 4$.

\subsection{Blazar SED modeling}

The aim of the SED modeling is twofold: 1- investigate the mechanism which produces the $\gamma$-ray emission; 2- constrain the jet power. We modeled the blazar SED using a onezone leptonic radiative model. In the model, the bulk of the jet emission is produced in a spherical region with radius $R$, in relativistic motion $\left(\Gamma_{b u l k}\right)$ at a distance, $z_{d}$ from the $\mathrm{SMBH}$. At low frequencies, the jet radiates via synchrotron mechanism. The high energy emission is produced via Compton scattering. Seed photons for the IC mechanism are the synchrotron emission in the blazar region and external radiation fields (synchrotron-selfCompton, SSC, and external Compton, EC, processes, respectively). External photons include the UV disk photons, the broad line region (BLR) photons and IR emission from the surrounding dust. The shape of the radiating electrons' energy distribution (EED) is a broken power-law and the spectral indexes below and above the energy break are derived from the observations. Following Ghisellini \& Tavecchio (2009), we made some standard assumptions on the geometry of the jet and seed photon regions and on the fraction of disk emission that is reprocessed by the BLRs and the dust. The total BLR luminosity $\left(L_{B L R} \sim 7 \times 10^{42} \mathrm{erg} \mathrm{s}^{-1}\right)$ was estimated from the $\mathrm{H}_{\alpha}$ line flux following Celotti et al. (1997).

The observed data are compatible with different combinations of the main model parameters. Specifically, we find that depending on the location of the emitting region, the 


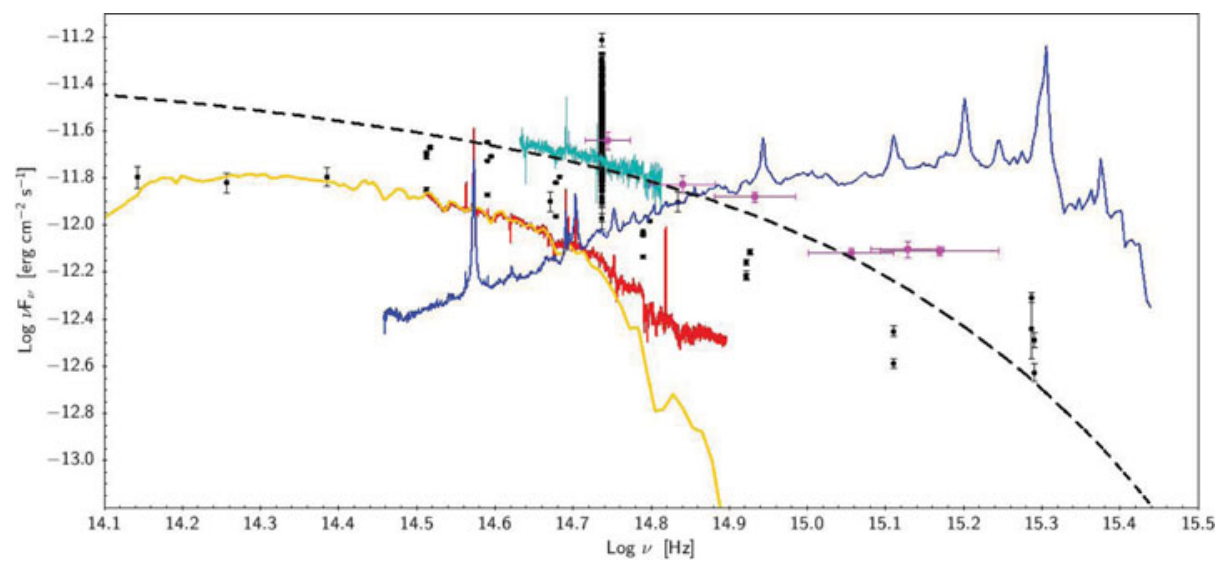

Figure 4. IR to UV SED of RGB J1512+020A core: the black points are the archival data retrieved from ASDC, the red line is the SDSS optical spectrum and the cyan line the spectrum of new Kitt Peak observation, the magenta points are the UVOT fluxes. The black dashed line is the synchrotron emission of the blazar region (see also Fig. 3), the yellow line is the host galaxy template of Mannucci et al. (2001) and the blue line is the quasar emission template of Vanden Berk et al. (2001).

bulk of the $\gamma$-ray emission could be due to EC either off BLR photons (in Fig. 3) or IR photons. The uncertainty on $z_{d}$, however, does not affect the estimated jet power (see the discussion in Sikora et al. 2009). We calculated $L_{j e t} \sim 10^{44}-10^{46} \mathrm{erg} \mathrm{s}^{-1}$, where the range accounts for uncertainties on the minimum Lorentz factor of the EED and the jet composition (i.e. the assumed number of cold protons per radiating electron, see Celotti \& Ghisellini 2008). The $L_{\text {jet }}$ inferred from the blazar SED modeling is in agreement with that derived from the total radio luminosity at $151 \mathrm{MHz}$ using the relation in Willott et al. (1999), $L_{j e t} \sim 10^{45} \mathrm{erg} \mathrm{s}^{-1}$. This would imply a jet that efficiently transports the bulk of the power to the radio lobes.

\subsection{Disk emission}

The near-IR (NIR) to optical-UV SED of quasar cores can be contributed to different levels by three main components: the non-thermal beamed jet emission, the thermal emission of the accretion disk and the host galaxy emission. All three contributions are present in the core spectrum of our source. In Fig 4, the black dashed line is the modeled blazar synchrotron obtained from the SED modeling. In low-z quasars, significant contamination of the $5100 \AA$ continuum from the host galaxy light is expected (see e.g. Shen et al. 2011). Indeed, the optical-NIR SED of RGB J1512+020A at wavelengths $\gtrsim 6000 \AA$ is nicely reproduced by the elliptical galaxy template (yellow continuum line) of Mannucci et al. (2001). Finally, for the quasar emission we adopted the composite optical spectrum (blue continuum line) of Vanden Berk et al. (2001), which accounts for the thermal continuum (big blue bump) and emission features (broad lines). The spectral modeling was done by adjusting the template to the observed continuum level and by matching the identified emission lines.

There is a clear discrepancy between the data and the quasar template, that appears to overpredict the observed emission in the UV band. The mismatch is even larger if we keep into account that part of the UV flux is due to synchrotron emission. Weak UV emission was previously reported by other authors (Wills \& Wills 1976 and Baldwin et al. 1989). Reddening due to flux absorption can be hardly reconciled with the observed broad line luminosity ratios (Baldwin et al. 1989). Furthermore, being a blazar, we expect a small 
inclination between the jet axis and the observer line of sight. Thus, it is likely that the UV disk emission is intrinsically weak. Assuming that the BLRs reprocess $\sim 10 \%$ of the disk luminosity $\left(L_{\text {disk }}\right)$, we calculated $L_{d i s k} \sim 7 \times 10^{43} \mathrm{erg} \mathrm{s}^{-1}$.

\section{Results}

Different estimates of the jet power of RGB J1512+020A indicates that $L_{\text {jet }}$ is at least comparable with or larger than the disk luminosity. If we assumed $L_{j e t} \sim 10^{45} \mathrm{erg} \mathrm{s}^{-1}$, as derived from the $151 \mathrm{MHz}$ radio luminosity, then $L_{j e t} \sim 10 L_{\text {disk }}$. This is in line with recent findings on Fermi blazars (Ghisellini et al. 2014), which would support a primary role of the magnetic field in the formation and launch of the jet. Interestingly, models of magnetically arrested accretion (Tchekhovskoy et al. 2011) predict a deficit of the UV flux of the disk due to the action of the magnetic field on the disk inner region (Sikora \& Begelman 2013), as observed in RGB J1512+020A.

\section{Acknowledgements}

The author thanks the International Astronomical Union for financial support.

\section{References}

Ackermann, M., et al. 2011,ApJ, 743, 171

Baldwin, J. A., et al. 1989, ApJ, 338, 630

Cara, M., et al. 2013, ApJ, 773, 186

Celotti, A. et al. 1997, MNRAS, 286, 415

Celotti, A. \& Ghisellini, G. 2008, MNRAS, 385, 283

Ghisellini, G. \& Tavecchio, F. 2009, MNRAS, 397, 985

Ghisellini, G., et al. 2014, Nature, 515, 376

Harris, D. E. \& Krawczynski, H. 2006, ARA\&A, 44, 463

Hogan, B. S., et al. 2011, ApJ, 730, 92

Jester, S., et al. 2007, MNRAS, 380, 828

Mannucci, F., et al. 2001, MNRAS, 326, 745

Marshall, H. L., et al. 2005, ApJS, 156, 13

Marshall, H. L., et al. 2011, ApJS, 193, 15

Massaro, F., Harris, D. E., \& Cheung, C. C., 2011, ApJS, 197, 24

Miller, B. P., et al. ApJ, 726, 20

Sambruna, R. M., et al. 2004, ApJ, 608, 698

Sambruna, R. M., et al. 2007, ApJ, 670, 74

Sbarrato, T., et al. 2012, MNRAS, 421, 1764

Shen, Y., et al. 2011, ApJS, 194, 45

Siebert, J., et al. 1998, MNRAS, 301, 261

Sikora, M., et al. 2009, ApJ, 704, 38

Sikora, M. \& Begelman, M. C. 2013, ApJL, 764, L24

Tavecchio, F., et al. 2007, ApJ, 662, 900

Tchekhovskoy, A., et al. 2011, MNRAS, 418, L79

Vanden Berk, D. E., et al. 2001, AJ, 122, 549

Willott, C. J., et al. 1999, MNRAS, 309, 1017

Wills, D. \& Wills, B. J. 1976, ApJS, 31, 143 